\title{
Expecting the unexpected, foreign body bronchus masquerading as subglottic stenosis: A Case Report
}

\author{
Deepak Paudel ${ }^{1}$, Raunak Dahal ${ }^{1}$, Bajarang Sah ${ }^{1}$, Shyam $_{\text {Chettri }}{ }^{1}$, and Pravid Gajurel ${ }^{1}$ \\ ${ }^{1}$ BP Koirala Institute of Health Sciences
}

June 23, 2021

\begin{abstract}
It is a rare event that a patient suspected to have a subglottic stenosis actually has a foreign body bronchus. We report a suspected case of subglottic stenosis secondary to partial hanging and prolonged intubation. However, surprisingly the rigid bronchoscopy revealed a betel nut at the level of carina.
\end{abstract}

\section{Introduction}

The subglottic region begins just below the vocal folds and extends to the lower border of the cricoid cartilage. In infants and young children, it is the narrowest section of the airway. The etiology of subglottic stenosis is congenital or acquired, with the latter encountered far more frequently. The most common cause of acquired subglottic stenosis is trauma, which can be internal (prolonged endotracheal intubation, tracheostomy, flame burn injury) or external (blunt or penetrating neck trauma). ${ }^{1,2}$ Acquired subglottic stenosis in a child can be a life-threatening situation requiring immediate airway management. It is rare for severe subglottic stenosis to be caused by an unexpected foreign body after airway securement following an external traumatic event in a child.

\section{Case History}

A thirteen years old girl presented to ENT OPD of BPKIHS with chief complaints of shortness of breath and throat pain for 4 days. Her throat pain was continuous and associated with difficulty in swallowing both solid and liquid food. One month back she had tried to commit suicide by partial hanging. Then she was taken to a local health centre and was intubated and kept in intensive care unit for 5 days. After extubation she again developed difficulty in breathing and was tracheostomized for further 20 days. Decannulation of tube was done 7 days prior to the presentation in our OPD.

On examination, she had noisy breathing with stridor. Furthermore, she had a stomal opening on the anterior neck of size $3 \times 1 \mathrm{~cm}^{2}$. She had increased respiratory rate with use of accessory muscle of respiration. We suspected her as a case of subglottic stenosis and admitted for further evaluation and kept under injectable antibiotics and corticosteroid. During her stay Computed Tomography (CT) scan of her neck was done and it showed 'a soft tissue attenuating thick band like structure causing luminal narrowing extending obliquely from the right posterolateral wall of subglottic portion of larynx to the left anterolateral wall of proximal trachea- likely granuloma/ inflammatory membrane' (Figure 1). We also did a psychiatry consultation and was diagnosed as 'Intentional Self Harm by hanging (impulsive) with borderline personality trait 'and was started on olanzapine.

\section{Investigations and treatment}

On the basis of history, examination and imaging the diagnosis of sub-glottic stenosis was made and for accurate pre-operative assessment of site, length and degree of stenosis, rigid bronchoscopy was done under 
general anesthesia. However, to our surprise a betel nut measuring $2 \times 1 \mathrm{~cm}^{2}$, covered with mucin, blackish debris and granulation tissues was found in the tracheal lumen just above the carina with no evidence of subglottic stenosis (Figure 2 and 3). The betel nut was then removed followed by disappearance of stridor and ease in breathing.

\section{Outcome and follow-up}

She was discharged on $2^{\text {nd }}$ postoperative day uneventfully and asked to remain on regular follow up. Repeat bronchoscopic evalution was done after 3 months which was normal. She is on regular follow up and has no complains related air way compromise and compliant over the medication prescribed by the psychiatrist.

\section{Discussion}

Foreign body aspiration (FBA) demands timely diagnosis and treatment, diagnostic evaluation of suspected FBA is based on a history of choking, cough, dyspnea, and decrease breathe sound, wheeze and stridor and among these signs and symptoms choking event is the most specific one. ${ }^{3}$ Most of the time history of choking or witness of the event is absent leading to delayed diagnosis and increasing the risk of complications. Study done by Karakoc et. al. revealed that inorganic foreign bodies are more common in adolescents. Due to radiopaque nature and positive history of aspiration these kind of foreign bodies are diagnosed promptly. On the other hand, organic FBA is associated with a longer elapsed time from aspiration to diagnosis with increased risk of complication like pneumonia and bronchiectasis, bronchial fistula, and it can even get secondarily dislodged and cause acute respiratory deterioration ${ }^{4,5}$

Our case was unique as the patient developed the shortness of breath after an attempt of partial hanging and intubation and tracheostomy to follow. This history compelled us to make the provisional diagnosis of subglottic stenosis without a second thought. Furthermore, CT scan findings also supported our diagnosis. The sensitivity of CT scan for the detection of bronchial FB is close to $100 \%$ with specificity between 66.7 and $100 \%$ and very few false-negative. Qiu et. al. found false negative rate of about $7 \%$ and it depended upon endobronchial inflammatory exudate, tracheobronchial stenosis or obstruction, which may cover up the sign of intrabronchial foreign body. When they were aspirated into the trachea or bronchus, they often located in parallel with the bronchial lumen, so the layer images of CT may miss the signs of FBs. ${ }^{6,7}$ In our case also the granulation tissue might have obscured the CT finding.

Airway stenosis due to long standing foreign body is underrated in the literature as only pneumonia, bronchiectesis and fistulas are described in most of the literatures. Any impacted FB causes mucosal trauma to airway lumen and initiate the granulation around it which then leads to cicatrization and airway stenosis. The process of stenosis may progress even after removing the foreign body signifying the need of periodic post-operative bronchoscopic evaluation. ${ }^{8}$

Patients suspected with sub glottis stenosis need accurate assessment for proper planning of the treatment and to prognosticate the future outcome. Though imaging is a simple, noninvasive and easily accessible tool for the assessment, rigid bronchoscopy is the basic assessment tool for precise pre-operative assessment of the site, grade and length of laryngotracheal stenosis. ${ }^{9}$ Similarly rigid bronchoscopy under general anesthesia is standard of care to diagnose and remove the air way foreign body. ${ }^{10}$

\section{Author's contribution:}

Author 1: Idea and preparation of manuscript

Author 2: Editing and literature review

Author 3: Diagnosis and intraoperative assistance

Author 4: Diagnosis and intraoperative assistance

Author 5: Diagnosis and intraoperative assistance

Acknowledgement 
I would like to thank Dr. Dibya Raj Mishra and Dr. Sabin Bhandari for their intraoperative assistance to manage the patient and Dr. Bibisha Baaniya for her help in making manuscript.

Conflict of interest : none

Funding Source : none

Consent statement: Patient provided written consent for publication of this case report.

Data availability : Data will be made available upon request

\section{References:}

1. Shott SR. Down syndrome: analysis of airway size and a guide for appropriate intubation. The Laryngoscope. 2000 Apr;110(4):585-92.

2. Blanchard M, Leboulanger N, Thierry B, Blancal J-P, Glynn F, Denoyelle F, et al. Management specificities of congenital laryngeal stenosis: external and endoscopic approaches. The Laryngoscope. 2014 Apr;124(4):1013-8.

3. Jung SY, Pae SY, Chung SM, Kim HS. Three-dimensional CT with virtual bronchoscopy: a useful modality for bronchial foreign bodies in pediatric patients. Eur Arch Otorhinolaryngol. 2012; 269(1):223-228. 1.

4. Karakoç F, Karadağ B, Akbenlioğlu C, Ersu R, Yildizeli B, Yüksel M, et al. Foreign body aspiration: what is the outcome? Pediatr Pulmonol. 2002 Jul;34(1):30-6.

5. Boufersaoui A, Smati L, Benhalla KN, Boukari R, Smail S, Anik K, et al. Foreign body aspiration in children: experience from 2624 patients. Int J Pediatr Otorhinolaryngol. 2013 Oct;77(10):1683-8.

6. Wu X, Wu L, Chen Z, Zhou Y. Fatal choking in infants and children treated in a pediatric intensive care unit: A 7- year experience. Int J Pediatr Otorhinolaryngol. 2018 Jul;110:67-9.

7. Qiu W, Wu L, Chen Z. Foreign body aspiration in children with negative multi-detector Computed Tomography results: Own experience during 2011-2018. Int J Pediatr Otorhinolaryngol. 2019 Sep;124:90-3.

8. Mehta RM, Rashmi N, Bajaj P, Krishnan S, Srinivasan L. Airway Stenosis Related to Foreign Body Aspiration: An Under-recognized Long term Complication. Clin Med Insights Case Rep. 2019;12:1179547619863816. Published 2019 Jul 19.

9. El-Naga HAA, El-Rasheedy AI, Abdelaziz M, Shawky M (2016). The Role of Multidetector CT Virtual Bronchoscopy in Assessment of Patients with Laryngotracheal Stenosis. J Otolaryngol ENT Res 5(2): 00135.

10. Fidkowski CW, Zheng H, Firth PG. The anesthetic considerations of tracheobronchial foreign bodies in children: a literature review of 12,979 cases. Anesth Analg. 2010 Oct;111(4):1016-25. 


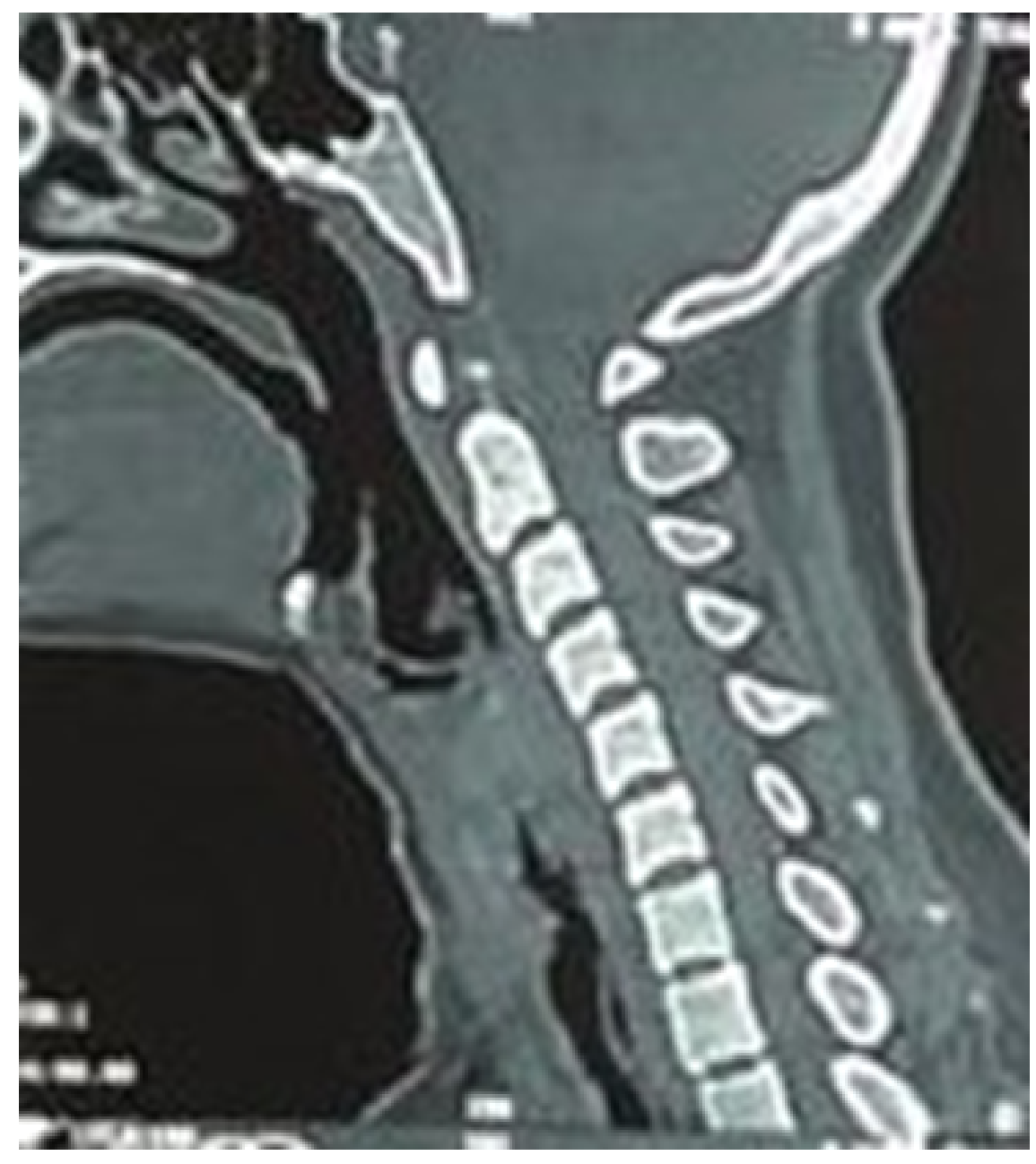



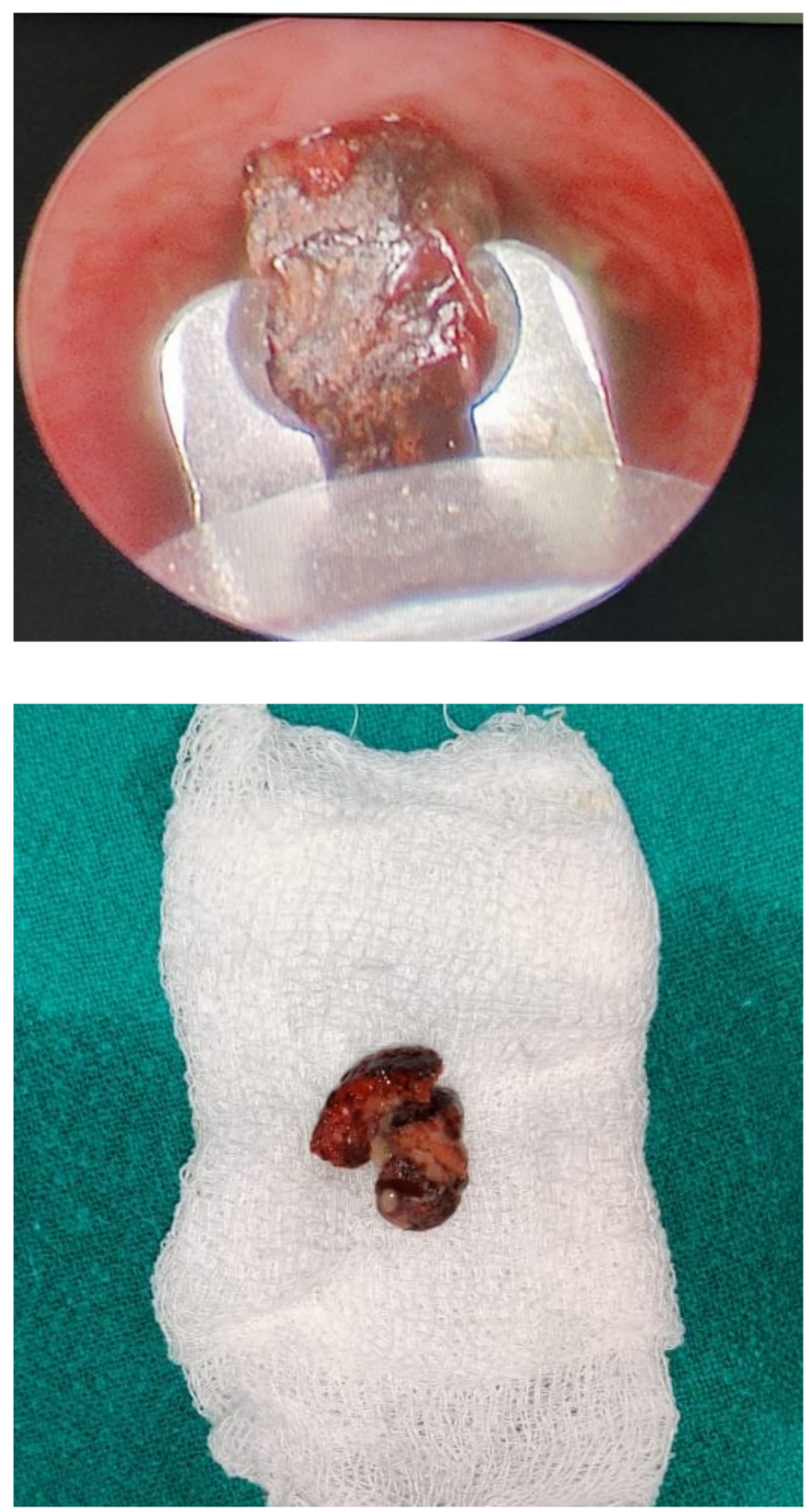